\title{
User Perceptions of 3D Food Printing Technologies
}

\section{Tom Gayler}

Lancaster University

Lancaster, LA1 4YW, UK

t.gayler@lancaster.ac.uk

\section{Prof. Corina Sas}

Lancaster University

Lancaster, LA1 4YW, UK

c.sas@lancaster.ac.uk

\section{Dr. Vaiva Kalnikaitè}

Dovetailed Ltd.

Cambridge, CB2 1NW

vaiva@dovetailed.io
Paste the appropriate copyright/license statement here. ACM now supports three different publication options:

- ACM copyright: ACM holds the copyright on the work. This is the historical approach.

- License: The author(s) retain copyright, but ACM receives an exclusive publication license.

- Open Access: The author(s) wish to pay for the work to be open Open Access: The author(s) wish to pay for the work
access. The additional fee must be paid to ACM.

This text field is large enough to hold the appropriate release statement assuming it is single-spaced in Verdana 7 point font. Please do not change the size of this text box.

Each submission will be assigned a unique DOI string to be included here

\begin{abstract}
3D food printing technologies offer a range of opportunities for $\mathrm{HCI}$, yet so far applications have been limited. We report a survey exploring the attitudes of early adopters towards 3D food printing technology, with the aiming of helping designers create successful applications for this technology.
\end{abstract}

\section{Author Keywords}

3D printing food technologies; user perceptions; technology adoption.

\section{ACM Classification Keywords}

H.5.m. Information interfaces and presentation (e.g., HCI): Miscellaneous.

\section{Introduction}

3D Food Printing (3DFP) is an emerging technology that offers a range of opportunities for designing novel user experiences. Food affords for multisensory experience and is deeply embedded in various social rituals in everyday life. Being able to leverage this connection provides interaction designers the opportunity to explore an emerging design space for user experience. As 3DFP technology finds new applications it requires consideration as to how its users perceive it. The relationship the user has with the technology has potential to impact 3DFP's adoption as well as its future 
applications. The main contributions of this paper include a comparison of food perception among different early adopter groups, and the perceived challenges and opportunities of 3DFP technologies.

\section{Literature Review}

We draw from HCI models of trust and technology adoption to approach the implications for 3DFP technology. Rogers [11] describes the characteristics of various user groups in relation to technology adoption. He identifies 'Early Adopters' as motivated by novel and rewarding experience and less concerned with the risks of technology in comparison to other population segments. The influence of risk and benefits on user has been explored through the Technology Adoption Model [12]. This model proposes 'Perceived Ease of Use' and 'Perceived Usefulness' as key factors influencing adoption. Ease of use is constructed from sources relating to the trust in, and experience of technology. These factors are balanced, receiving different weights from differing populations. From the perspective of food technologies, both risks [2] and trust [5] are reported as playing a role in influencing consumer acceptance of a foodstuff.

The interplay of the above factors has been applied to autonomous vehicles [10], with findings showing that positive user experiences best support adoption. The challenge of understanding the risks and benefits of 3DFP technologies lies in the lack of concrete applications. In order to create 3DFP technologies that are successfully adopted, it is important to understand perceived risks and envisaged contexts of use, in particular amongst early adopters who shape wider consumer perception [11].

\begin{tabular}{c|c|c|c|c|c} 
Sample & $\mathbf{N}$ & Mean & s.d. & z-test & Sig. (p) \\
\hline $\begin{array}{c}\text { Cox \& Evans } \\
\text { Population } \\
\text { [3] }\end{array}$ & 294 & 55.00 & 11.90 & -- & -- \\
\hline $\begin{array}{c}\text { 3DFP Mailing } \\
\text { List - [6] }\end{array}$ & 24 & 41.54 & 12.00 & -5.54 & $<0.01^{*}$ \\
\hline $\begin{array}{c}\text { 3D Printing } \\
\text { Forums }\end{array}$ & 6 & 40.83 & 10.36 & -2.92 & $<0.01^{*}$ \\
\hline $\begin{array}{c}\text { Computer } \\
\text { Science } \\
\text { Students }\end{array}$ & 20 & 48.15 & 8.31 & -2.57 & $<0.01^{*}$
\end{tabular}

Table 1: Food Technology Neophobia Scores of survey groups and scale validation population. Significant difference is reported for each study group in comparison to population from [3].

The potential of 3DFP printing within HCI has received limited attention; a noticeable exception being the EdiPulse system, that prints chocolate rewards for physical activity [8]. With respect to the perception of 3DFP technology, we previously reported a preliminary analysis of survey data from 24 users of 3DFP, with findings showing the experimental quality of such technologies and people's knowledge of sugary and fatty foodstuff [6]. The current paper extends this prior work in two ways by reporting data from 50 survey participants from two additional communities, and their richer analysis.

\section{Method}

The research method consists of a survey exploring early adopters' perception, attitudes and knowledge of 3DFP technologies, which consisted of three parts.

First, we used two valid and reliable scales, i.e. Food Technology Neophobia Scale (FTNS) [3], and Social Representations of Scale for novel foods [9] 


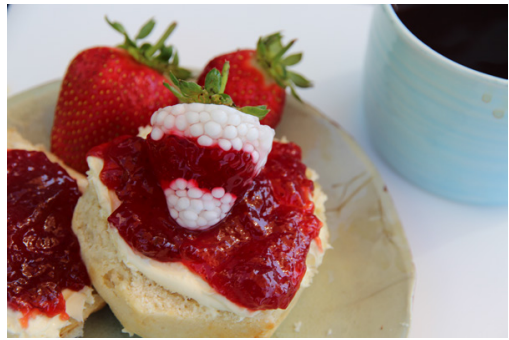

Figure $1: 3 \mathrm{D}$ printed strawberry and cream berry. This demonstrates how foodstuffs can be reimagined through 3DFP technology. Photo courtesy of Dovetailed Ltd.

\begin{tabular}{|c|c|c|c|c|c|c|c|c|c|c|c|c|c|c|c|}
\hline \multirow{2}{*}{ Sample } & \multicolumn{3}{|c|}{$\begin{array}{c}\text { Adherence to } \\
\text { Technology }\end{array}$} & \multicolumn{3}{|c|}{$\begin{array}{l}\text { Adherence to } \\
\text { Natural Food }\end{array}$} & \multicolumn{3}{|c|}{ Enjoyment } & \multicolumn{3}{|c|}{ Necessity } & \multicolumn{3}{|c|}{ Suspicion } \\
\hline & $M$ & s.d. & z-test & $M$ & s.d. & z-test & $M$ & s.d. & z-test & $M$ & s.d. & z-test & $M$ & s.d. & z-test \\
\hline $\begin{array}{l}\text { Onwezen \& } \\
\text { Bartels [9] }\end{array}$ & 3.11 & 0.80 & -- & 3.75 & 0.88 & -- & 3.70 & 0.84 & -- & 2.20 & 0.87 & -- & 3.01 & 0.73 & -- \\
\hline $\begin{array}{l}\text { 3DFP Mailing } \\
\text { List }\end{array}$ & 5.21 & 0.87 & $12.86 *$ & 5.25 & 1.25 & $8.35 *$ & 6.22 & 0.77 & $\begin{array}{c}14.70 \\
*\end{array}$ & 2.57 & 1.06 & 2.08 & 3.87 & 0.90 & $5.57^{*}$ \\
\hline $\begin{array}{l}\text { 3D Printing } \\
\text { Forum }\end{array}$ & 5.17 & 0.87 & $6.31 *$ & 3.67 & 1.61 & $3.95 *$ & 5.50 & 0.84 & $5.25 *$ & 3.00 & 0.87 & 2.25 & 3.67 & 1.48 & 2.21 \\
\hline $\begin{array}{l}\text { Computer } \\
\text { Science } \\
\text { Students }\end{array}$ & 4.77 & 0.85 & $9.28 *$ & 4.40 & 1.65 & $5.18^{*}$ & 5.70 & 1.42 & $\begin{array}{c}10.65 \\
*\end{array}$ & 3.27 & 1.47 & $5.50 *$ & 3.90 & 0.95 & $5.45^{*}$ \\
\hline
\end{tabular}

Table 2 : This table reports the scores for the three groups on the Social Representation Scale in comparison to the population scores from the validation study. Z-tests where $p<0.01$ are marked with a $*$

developed by food science researchers for measuring consumers' perception of novel foodstuffs. Second, we asked participants to report their perceived risks for sourcing, processing, selling, preparing and eating 3D printed food. Third, we also asked about direct experiences of 3D food printing and the envisaged contexts where participants expected to see this technology used in.

\section{Participants}

The survey was targeted to three communities of potential early adopters' and answered by 24 members of Nufood's 3DFP mailing list, 6 members of a 3D printing forum (www.3dprintboard.com), and 20 computer science undergraduate students at Lancaster University. This study explores how well these groups do in fact, represent early adopters.

\section{Results}

We now report the survey findings focusing on the attitudes, perceived risks and envisaged contexts of use of $3 \mathrm{D}$ printing technologies, alongside their comparison across the three groups.

Attitudes toward 3D Printing Food Technologies Table 1 and 2 show the Mean and Standard deviation for each of the three groups on the two scales, alongside with the population score used for validating the scales $[3,9] . Z$ tests indicate that all groups are less neophobic towards food technology than the original population used; 3DFP Mailing List $(z=-5.54$, $p<0.01)$; 3D printing forum $(z=-2.92, p<0.01)$ Computer Science students $(z=-2.57, p<0.01)$. $Z$ tests for Social Representation Scale presented in Table 2 also indicate that all three groups show significantly higher adherence to technology than the validating 


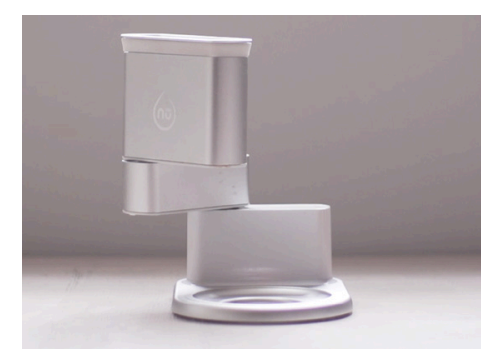

Figure 2: Nufood printer. This designed to fit on a tabletop allowing for 3DFP to take place beyond industry and lab contexts. Photo courtesy of Dovetailed Ltd. population [9]; 3DFP Mailing List $(z=-12.86, p<0.01)$; $3 \mathrm{D}$ printing forum $(z=6.31, p<0.01)$; Computer Science students $(z=9.28, p<0.01)$.

A comparison of these scale' scores across the three groups shows significant differences on the enjoyment dimension (3DFP Mailing List $z=14.70, p<0.01$; 3D printing forum $z=5.25, p<0.01$; Computer Science students $z=10.65, p<0.01$. This suggests that exposure or interest in 3D food printing may offer a more engaging experience as opposed to 3D printing in general. Together these findings indicate that all three groups are significantly more open towards engaging with the novel 3D printing food technologies which is the marker of early adopters, with adopters of 3D printing food technology being more engaged than other groups.

Perceived Risks of 3D Food Printing

Food technologies bring a new set of considerations for consumers deciding to try a novel foodstuff [3]. To better understand how such risks of 3D food printing differ from other food technologies we prompted participants to describe perceived risks at five stages of food production (sourcing raw materials, processing materials into products, selling products, preparing products for consumption and eating the product).

Findings indicate that perceived risks of sourcing 3D printed food is centered on environmental concerns, in line with the environmental impact of production for other food technologies $(\mathrm{n}=9)$ : "will [it] require the same land as non-printed food? No environmental benefit would make this more of a fad" [Participant 18, Student]. When asked to consider the processing of this food, the major risks were adulteration and additives included in the final food product: "I imagine a number of non-nutritional preservatives will possibly have to be added to the food" [P12, Student] In contrast to

product-focused risks for the sourcing and processing, the perceived risk for selling related to consumers' lack of awareness and bias against this technology:

"consumer bias against trying new things and the stigma of 'artificial' food" [P8, Student].

Perceived risks related to the preparation are where the most prominent divergence from traditional foodstuffs occurs. The major issue was the misuse or malfunction of the printer $(n=20)$. Unlike most other food technologies (GM, Mass Production) the technology of 3D printed food is located much closer to the consumer. The potential for more creative use of the foodstuff is placed in the hands of the consumer but it also shifts the risk of malfunction closer to the consumer as well. The most prominent eating risk related to health and diet $(n=14)$ however it was more nuanced with a focus on "long term negative effects [that] are unknown, and untestable." [P7, Student]. This uncertainty suggests a challenge in moving 3D food printing technology from a one-off experience into a tool for every-day use in domestic contexts.

While the risks we report at each stage echo the commonly perceived risks for all food technologies [2] they also focus on technology's common myths. In the selling of food, the major concerns related to its opacity, limiting people's ability to understand how it works. There is no existing mental model for making sense of this technology which if not addressed, could hinder adoption. 
Envisaged Uses of 3D Food Printing

The most commonly known 3D printed foodstuffs are predominantly sweet tasting, chocolate being the most used [8]. Sugar was another commonly mentioned foodstuff also used in 3D System's Culinary Lab [1]. When asked if they had actually tried 3D printed food (3DFP Mailing list $n=10$, 3D Printing Forum $n=1$, Computer Science Students $n=1$ ) they reported trying it at exhibitions or dining events. In line with this being an emerging technology it is expected to be seen at exhibitions but its use in dining events suggests the technology may first mature as a tool whose primary purpose is provision of experience, rather than the provision of nourishment.

When asked about the envisaged uses for 3D food printing technology most participants mentioned research $(n=22)$, This suggests a non-domestic application, and a still uncertain or undefined place for 3DFP. The second most envisaged use was for creative purposes $(n=21)$, here the ability of 3DFP to create "novel shapes for confectionary" [P18, Mailing List] was understood to offer potential for application in decoration and presentation of food. A more surprising use for the technology was in the provision of food aid in emergency situations $(n=7)$. None of the participants provided a reasoning for this, although it is possible the technology is perceived as being able to construct satisfying meals from limited resources, we can see some evidence for this claim in participant 19's [Student] potential use of the technology to "convert rotten food to edible foods". A perhaps more feasible expectation for 3DFP is in assisting healthcare and healthy eating $(n=6)$. Expectations ranged from portion control (recognizing the influence of digital control) to preparation of personalized diets (recognizing the model of micro-manufacture offered by 3D printing).

We also prompted speculative responses from our participants to imagine more unusual applications for 3DFP. Personalization $(n=6)$ was the most often mentioned context, suggesting consumers' desire to tailor meals or dining experiences to their tastes. This also prompted responses that looked beyond the dinner plate with edible packaging [P15, Student], edible tattoos [P11, Student] and even sex toys [P14, Mailing List].

\section{Discussion}

From our results we can see that 3DFP technology is mainly understood as a non-domestic technology and with concerns over the long term effects to health from the foodstuff produced, it seems there is reassurance needed. Our early adopters were shown to be highly influenced by the enjoyment provided by food technology and this suggests that like Rödel et al. [10] focus should be on creating contexts of use in which the user experience is prioritized. In their speculations participants reported personalization of their meals with 3DFP as a potential use. This offers an exciting opportunity to engage users in the design of their own food experiences, allowing them to control parameters such as taste and form with the production of their design handled by the printing technology.

It is clear that early adopters are inspired by 3DFP's potential to be used in emergency aid contexts. Whilst there remain questions over the usefulness of 3DFP to this end, exploration in this area will be welcomed and supported by early adopters. Our findings suggest that such a humanitarian or entertainment value should be 
added to 3DFP in order to drive awareness and familiarity with the technology. 3DFP robots allow the production of food to take place in front of users eyes. There is an opportunity to consider how the movements and process of printing can be designed into rituals of 3D printed food consumption.

Regardless of the specific application we have seen that lack of awareness amongst consumers is providing resistance to adoption in everyday contexts. As 3DFP technology matures further work will need to be undertaken to communicate the process and value of the technology to users. If education about 3DFP is paired with an engaging application then it has the potential to be a widely adopted and successful technology.

\section{Acknowledgements}

This work was supported by the Designing Multisensory Interactions: Emotional User Experience of 3D PrintedFood Consumption project funded by EPSRC and Dovetailed Ltd. under a CASE award.

\section{References}

1. 3DS Culinary Labs. 2015. 3DS Culinary. 3D Systems. Retrieved October 17, 2016 from https://www.3dsystems.com/es/culinary.

2. Angela Bearth and Michael Siegrist. 2016. Are risk or benefit perceptions more important for public acceptance of innovative food technologies: A meta-analysis. Trends in Food Science \& Technology 49: 14-23.

3. D. N. Cox and G. Evans. 2008. Construction and validation of a psychometric scale to measure consumers' fears of novel food technologies: The food technology neophobia scale. Food Quality and Preference 19, 8: 704-710.

4. Lynn Frewer. 1999. Risk Perception, Social Trust, and Public Participation in Strategic Decision Making: Implications for Emerging Technologies. Ambio 28, 6: 569-574.

5. Tom Gayler. 2017. Towards Edible Interfaces: Designing Interactions with Food. Proceedings of the 19th ACM International Conference on Multimodal Interaction, ACM, 623-627.

6. Rohit Ashok Khot, Deepti Aggarwal, Ryan Pennings, Larissa Hjorth, and Florian "Floyd" Mueller. 2017. EdiPulse: Investigating a Playful Approach to Self-monitoring Through 3D Printed Chocolate Treats. Proceedings of the 2017 CHI Conference on Human Factors in Computing Systems, ACM, 6593-6607.

7. Marleen C. Onwezen and Jos Bartels. 2013. Development and cross-cultural validation of a shortened social representations scale of new foods. Food Quality and Preference 28, 1: 226234.

8. Christina Rödel, Susanne Stadler, Alexander Meschtscherjakov, and Manfred Tscheligi. 2014. Towards Autonomous Cars: The Effect of Autonomy Levels on Acceptance and User Experience. Proceedings of the 6th International Conference on Automotive User Interfaces and Interactive Vehicular Applications, ACM, 11:1$11: 8$.

9. Everett M. Rogers. 2003. Diffusion of Innovations, 5th Edition. Free Press, New York.

10. Viswanath Venkatesh and Hillol Bala. 2008. Technology Acceptance Model 3 and a Research Agenda on Interventions. Decision Sciences 39, 2: 273-315. 